\title{
Gas cells for tunable diode laser absorption spectroscopy employing optical diffusers. Part 2: Integrating spheres.
}

\author{
D Masiyano $^{1,2}$, J Hodgkinson ${ }^{1} *$ and R P Tatam ${ }^{1}$ \\ 1 Engineering Photonics Group, School of Engineering, Cranfield University, Bedfordshire, \\ MK43 0AL, UK. \\ 2 Now at: Alps Electric (UK) Ltd, Garamonde Drive, Wymbush, Milton Keynes, MK8 8LW, UK. \\ * corresponding author j.hodgkinson@cranfield.ac.uk
}

\begin{abstract}
We have studied the effects of random laser speckle and self-mixing interference on TDLS based gas measurements made using integrating spheres. Details of the theory and TDLS apparatus are given in Part 1 of this paper and applied here to integrating spheres. Experiments have been performed using two commercial integrating spheres with diameters of $50 \mathrm{~mm}$ and $100 \mathrm{~mm}$ for the detection of methane at $1651 \mathrm{~nm}$. We have calculated the expected levels of laser speckle related uncertainty, considered to be the fundamental limiting noise, and imaged subjective laser speckle in a sphere using different sized apertures. For wavelength modulation spectroscopy, noise equivalent absorbances (NEAs) of around $5 \times 10^{-5}$ were demonstrated in both cases, corresponding to limits of detection of $1.2 \mathrm{ppm}$ methane and $0.4 \mathrm{ppm}$ methane respectively. Longer-term drift was found to be at an NEA of $4 \times 10^{-4}$. This lies within our broad range of expectations. For a direct spectral scan with no wavelength dither, a limit of detection of $75 \mathrm{ppm}$ or fractional measured power uncertainty of $3 \times 10^{-3}$ corresponded well with our prediction for the objective speckle uncertainty.
\end{abstract}

\section{PACS codes}

07.07.Df sensors - chemical

42.62.Fi laser spectroscopy

42.30.Ms Speckle and moire patterns

42.79.-e optical instruments, equipment and techniques 


\section{Introduction}

Multi-pass cells are often used in tunable diode laser spectroscopy (TDLS) in order to increase the optical pathlength of the system and thereby improve limits of detection. Examples include designs by White ${ }^{[1]}$, Herriott $^{[2]}$ and Chernin ${ }^{[3]}$. Essentially in each case a collimated beam of light is reflected a number of times between two mirrors to give a total pathlength that can be a considerable multiplier of the physical length of the cell. For example, a commercial Herriott cell design folds a $30 \mathrm{~m}$ beam into a physical length of $40.5 \mathrm{~cm}^{[4]}$.

A second approach to multi-pass cells is to allow overlap of consecutive passes of the light beam across the cell and either integrate the total output (in the case of integrated cavity output spectroscopy, ICOS, or cavity enhanced absorption spectroscopy ${ }^{[5]}$ ) or to work in the time domain and measure the decay time of pulses in the cavity (in the case of cavity ringdown spectroscopy, $\operatorname{CRDS}^{[6]}$ ). Equivalent pathlengths for these systems are the longest currently available (eg $10 \mathrm{~km}$ path in a cavity of physical length $1 \mathrm{~m}^{[6]}$ ), resulting from the use of high quality dielectric mirrors. Confocal designs ease the alignment problem, such that these very long pathlengths may be aligned with care, but again this is a time-consuming process.

Each design has its advantages, and systems may be optimised for improved pathlengths, greater tolerance to cell bending during use, overlap between adjacent reflected beams and so on ${ }^{[7]}$. However, all suffer from the problem of difficult alignment, which is exacerbated by the need to also misalign potential optical paths through etalons and sources of feedback to the laser diode, in order to minimise interference fringes in the system. Such fringes are often the performance - limiting source of uncertainty in multi-pass cells (as indeed they can be in single pass cells) ${ }^{[8]}$. Multi-pass cells can be time-consuming to align, and mechanical tolerances for field use are tight.

It is for these reasons that interest has grown in the use of integrating spheres as gas cells. The internal walls of the sphere have very high levels of reflectance (up to 99\%), but this is diffuse - the light is scattered in all directions. Good quality commercial spheres aim to use perfectly Lambertian reflectors, such that the radiance $L$ (in $\mathrm{W} \mathrm{m}^{-2} \mathrm{sr}^{-1}$ ) from a given point is a constant in any direction. For a cavity with spherical geometry, it follows that the irradiance $E$ (in $\mathrm{W} \mathrm{m}^{-2}$ ) received from that point is then constant over the entire sphere ${ }^{[9,10]}$. In the ideal case, after a single pass the irradiance at the sphere walls is perfectly uniform over the surface. 
The advantages are as follows. Alignment tolerances are relaxed and absorption measurements may be made on turbid or irregular samples. Commercial integrating spheres are available as accessories for use with FTIR spectrometers ${ }^{[11]}$. For measurements using broadband emitters such as LEDs and filtered incandescent or discharge lamps, Elterman ${ }^{[10]}$ has demonstrated measurement of the absorption of glass samples, Fry et al. ${ }^{[12]}$ have measured visible absorption in aqueous solutions, Fecht and Johnson ${ }^{[13]}$ have adapted a sphere for measurements on falling aqueous sample streams, and Hodgkinson et al. ${ }^{[14]}$ have adapted a closed photothermal cell by inserting an integrating cavity. Work at the University of Limerick has resulted in broadband spectroscopic sensors for $\mathrm{CO}_{2}$ (at $1.57 \mu \mathrm{m}$ and $2 \mu \mathrm{m}$ ) ${ }^{[15]}$ and $\mathrm{NO}_{2}$ and $\mathrm{SO}_{2}$ (in the UV/visible $)^{[16]}$.

The use of integrating spheres in TDLS has long been of interest. To our knowledge, Venkatesh et al. ${ }^{[17]}$ were first to use an integrating sphere, monitoring carbon monoxide around $5 \mu \mathrm{m}$. Abdullin and Lebedev ${ }^{[18]}$ have used a copper sphere with a $\mathrm{CO}_{2}$ laser to measure ammonia, and Tranchart et al. ${ }^{[19]}$ have used a $10 \mathrm{~cm}$ diameter sphere at $830 \mathrm{~nm}$ and $1.2 \mu \mathrm{m}$ to detect water vapour and butane respectively. These studies confirmed that integrating spheres offer increased pathlengths within a relatively small volume without the need for precise alignment. However, the performance limitations of these systems have not been established. In Part 1 of this paper, we applied our methodology for the study of diffuse reflections in TDLS $^{[20]}$ to the use of optical diffusers in simple, single and dual pass cells. In part 2, we now apply this understanding to the use of integrating spheres as multipass cells, with particular attention to the behaviour of random laser speckle and self-mixing interference.

\section{Theory}

A complete description of the speckle theory used here is given in Part 1 of this paper. Here, we concentrate on the specifics of the use of integrating spheres. Figure 1 shows a simplified model of the integrating sphere used in our experiments.

Tranchart et al. ${ }^{[19]}$ have shown that for an integrating sphere of internal radius $R$ and high values of mean surface reflectivity $\rho$, the radiant flux (in Watts) $\Phi$ for low $\alpha z(\alpha z \ll 1)$ and high $\rho((1-\rho) \ll 1)$ is approximated by;

$$
\frac{\Delta \Phi}{\Phi_{0}} \approx \frac{\alpha z_{0}}{(1-\rho)}
$$


where $\Phi_{0}$ is the radiant flux in the absence of gas, $\Delta \Phi$ is the change in radiant flux $\left(\Phi-\Phi_{0}\right)$ and the mean pathlength for a single pass is $z_{0}=\frac{4}{3} R$. They then derive the effective total pathlength for low or zero absorbance $\alpha$ within the sphere, $Z_{\text {eff; }}$;

$$
Z_{\text {eff }} \approx \frac{4}{3} \frac{R}{(1-\rho)}
$$

We have shown that in the general case of higher values of $\alpha$ and lower $\rho$, the following expression applies $^{[21]:}$

$$
\frac{\Phi}{\Phi_{0}}=\frac{\ln \rho}{\ln \rho-\alpha z_{0}}
$$

The throughput of the sphere is defined as the ratio of light collected at the detector to that entering the sphere, and is given by ${ }^{[19]}$

$$
\frac{\Phi_{\text {exit }}}{\Phi_{\text {in }}}=\frac{\rho f_{\text {exit }}}{1-\rho\left(1-f_{\text {total }}\right)}
$$

where $\Phi_{\text {exit }}$ is total flux exiting a given port (Watts); $\Phi_{\text {in }}$ is total incident flux (Watts), $f_{\text {exit }}$ is exit port fraction (area of exit port /surface area of sphere), $f_{\text {total }}$ is total port fraction (sum of all port areas/surface area of sphere), and $\rho$ is the sphere wall reflectance $(0 \leq \rho \leq 1)$.

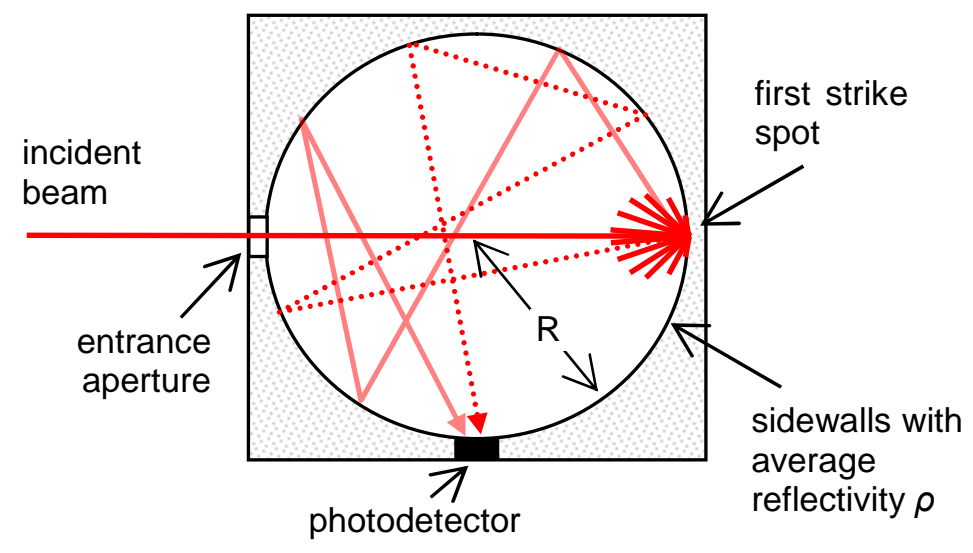

Figure 1. Simplified model of an integrating sphere, showing a collimated beam making a first pass across the cell to the first strike spot. Two examples are shown of light beams making subsequent random multiple passes across the cell.

Note that both the equivalent pathlength and the throughput are sensitive functions of the mean surface 
reflectivity, $\rho$. This parameter is governed by the material reflectivity at the wavelength of operation and by the proportion of non-reflective area taken up by, for example, the entrance aperture, photodetector and housing, plus gas inlet and outlet ports. It is not usually sufficient to calculate $\rho$ by using manufacturer's reflectivity data and knowledge of the cell geometry; instead, cell calibration is required using equations (1) or (3). This calibration needs to be completed whenever there is a change to the optical set-up inside the sphere, or if in field use, it would need to be completed with sufficient frequency to prevent any buildup of dirt inside the sphere from causing the pathlength to drop by enough to render the instrument specification invalid.

\subsection{Random laser speckle}

Using equations (4)-(6) of Part 1, we can evaluate the uncertainty resulting from random laser speckle as $\Delta \Phi / \Phi_{0}$ for both objective and subjective speckle, by making some assumptions about the cell geometry. For our integrating sphere, the lensless detector has a limited field of view $\left( \pm 30^{\circ}\right)$ that defines the illumination geometry of equation (4), Part 1 . If we were to use a high NA lens in front of the detector, we would evaluate equation (5), Part 1 assuming $L / a \sim 1$. Note that, for each type of speckle, the results are independent of the diameter of the sphere. The results are shown in Table 1, and in this case show the same expected level of uncertainty in the measurement of $\Delta \Phi / \Phi_{0}$.

Table 1. Estimated uncertainties in detected signals for random laser speckle

\begin{tabular}{|l|c|c|}
\hline Type of speckle & $\begin{array}{c}\text { Estimated } \\
\text { uncertainty in } \Delta \Phi / \Phi_{0}\end{array}$ & $\begin{array}{c}\text { Estimated change in } \Delta \Phi / \Phi_{0} \\
\underline{\text { under wavelength modulation }}\end{array}$ \\
\hline Objective speckle (no lens) $^{\mathrm{a}}$ & $2 \times 10^{-3}$ & $2 \times 10^{-3}-2 \times 10^{-7}$ \\
\hline Subjective speckle (with lens) $^{\mathrm{b}}$ & $2 \times 10^{-3}$ & $2 \times 10^{-3}-2 \times 10^{-7}$ \\
\hline
\end{tabular}

a $\pm 30^{\circ}$ field of view, $\lambda=1651 \mathrm{~nm}, D=1 \mathrm{~mm}$
b $L / a \sim 1, \lambda=1651 \mathrm{~nm}, D=1 \mathrm{~mm}$
c $\quad \Delta \lambda=0.025 \mathrm{~nm}, h \geq \lambda$

Now consider the effect of wavelength modulation using equation (9) of Part 1. For $h \sim \lambda$ and $\delta \lambda \sim 0.025 \mathrm{~nm}$, the uncertainty is reduced by a factor of $10^{4}$, and for higher values of $h$ the improvement is between this value and unity (no improvement). The resulting expected uncertainty is also given in Table 1. 
Integrating spheres developed for the mid IR use roughened surfaces coated with gold. For single scattering from this surface, the value of $h$ would be equal to twice the RMS deviation of the roughened surface. However, spheres designed for use in the visible and near IR typically use proprietary sintered poly [tetrafluoroethylene] (PFTE) materials known as Spectralon ${ }^{\mathrm{TM}}$ or Zenith ${ }^{\mathrm{TM}}$. These are multiple scatterers, in which light penetrates some distance into the bulk (up to $10 \mathrm{~mm}$ ) before re-emerging in the backscattered direction. These materials produce well-developed, high contrast speckle fields, but the effective value of $h$ is unknown. Therefore we cannot predict the expected speckle-related uncertainty with any precision; the range of expectation in Table 1 is very broad, namely 4 orders of magnitude.

It should also be noted that the theory above has not taken into account the effects of depolarisation of the light. Multiply scattered light from an integrating sphere is expected to be fully depolarised, and light backscattered from a bulk scattering material such as Spectralon or Zenith may be partially depolarised, depending on the material thickness. Full depolarisation is expected to result in the creation of two independent speckle fields at orthogonal polarisation states ${ }^{[22]}$, which would further reduce the speckle uncertainty by a factor of $\sqrt{ } 2$.

\subsection{Other interferometric effects}

Interferometric speckle is described in Part 1, section 2.3. However, for an integrating sphere geometry as shown in Figure 1 we have disrupted any possible return path of a reference beam. As long as there is no direct line of sight from the entrance aperture window to detector, we do not expect to encounter interferometric speckle effects when using integrating spheres.

Self-mixing or feedback interference fringes can arise when low levels of light are backscattered to the laser diode, as described in Part 1, section 2.4. The first strike spot is the main source of feedback to the laser diode. Our experiments (see below) have been designed to minimise this feedback, which is particularly troublesome when using backscattering materials.

\section{Experimental details}

The details of our experiments are similar to those described in Part 1, with the exception of the integrating sphere itself. Figure 2 shows a schematic diagram. 


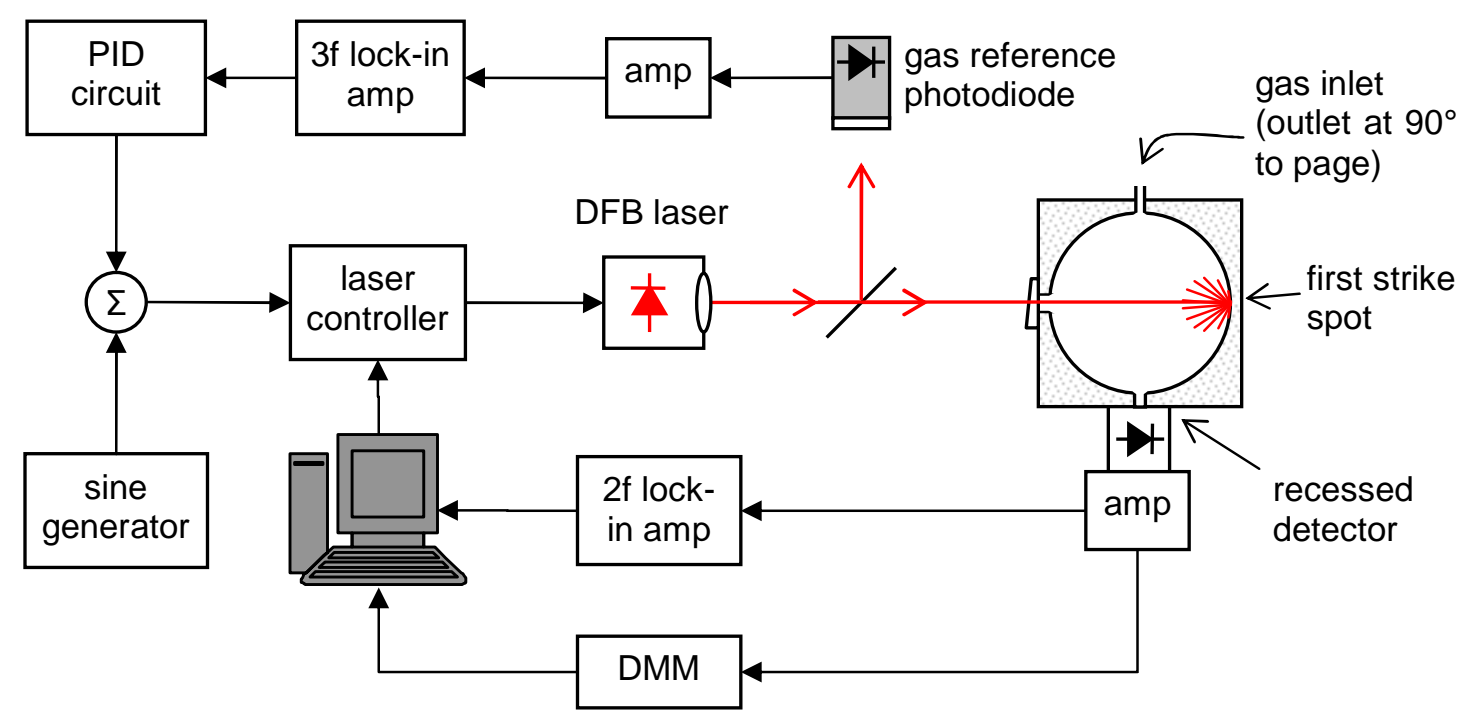

Figure 2. Schematic experimental configuration for $2 \mathrm{f}$ wavelength modulation spectroscopy, using a $3 \mathrm{f}$ line lock.

Two integrating spheres were used, both incorporating Zenith ${ }^{\mathrm{TM}}$ as the reflective material. The first (Thorlabs IS200-4, manufactured by Sphere Optics) had an internal diameter of $50.8 \mathrm{~mm}$; for simplicity we term this the "50 mm sphere". Light from the laser diode entered the integrating sphere via a $6.35 \mathrm{~mm}$ diameter entry port covered by an angled and antireflection coated window (Thorlabs PS812-C). The detector (12.5 mm diameter port, Thorlabs PDA400) was recessed to prevent a direct line of sight light from the first strike spot, therefore no baffle was necessary. The detector's field of view was restricted to $\pm 32^{\circ}$. Two additional ports $(1.5 \mathrm{~mm}$ diameter $)$ allowed entry and exit of test gas mixtures. The second integrating sphere (Sphere Optics SPH-4Z-4) had an internal diameter of $101.6 \mathrm{~mm}$ (termed the "100 mm sphere") and a similar port arrangement, except that in this case the detector was not recessed and an internal baffle was present. We employed $6 \mathrm{~mm}$ diameter ports for the inlet aperture and detector, and $1.5 \mathrm{~mm}$ diameter ports for the gas inlet and outlet. In a previous publication, we have shown that the equivalent pathlength of the $50 \mathrm{~mm}$ and $100 \mathrm{~mm}$ spheres, in the absence of gas, was $1.2 \mathrm{~m}$ and $3.4 \mathrm{~m}$ respectively ${ }^{[21]}$.

\section{Results}

\subsection{Speckle behaviour}

To confirm the behaviour of random laser speckle inside the $50 \mathrm{~mm}$ diameter sphere, we took images 
through an open sphere detector port using a silicon CCD camera (Pearpoint P176) and illumination via an entrance port at $90^{\circ}$ from a HeNe laser. As shown in Figure 3, images were taken using a lens placed at the exit port, with the CCD chip at a further distance of 50mm; this geometry decreased the NA and should therefore exaggerate the speckle size.

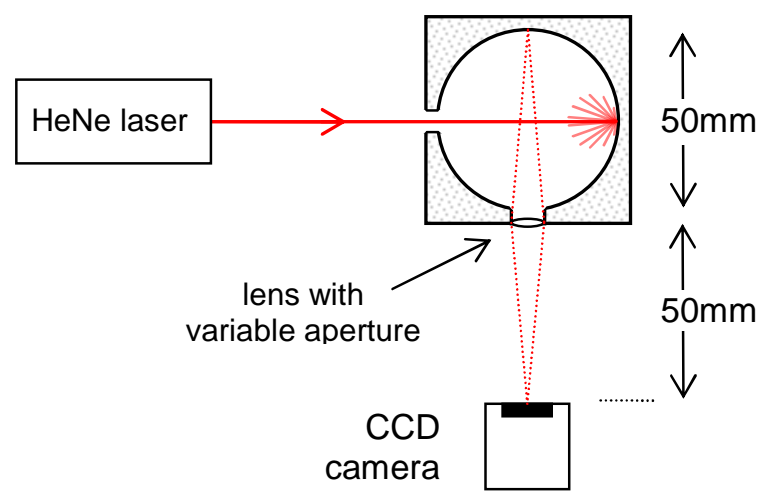

Figure 3. Experiment to image subjective speckle in an integrating sphere.

Figure 4 shows the resulting speckle images taken with different sized lens apertures. Also shown in Figure 4 are the speckle sizes predicted using equation (5), Part 1 for $L=50 \mathrm{~mm}$ and $\lambda=633 \mathrm{~nm}$. Limited resolution of the images means that only the speckles in Figure 4 (d) are fully resolved, therefore images (a) - (c) suffer some loss of contrast to differing degrees. The dark vertical line in the images corresponds to a construction feature of the integrating sphere - the join between two hemispheres. 
(a) a=12.7mm, $\varepsilon_{\mathrm{s}}=3 \mu \mathrm{m}$

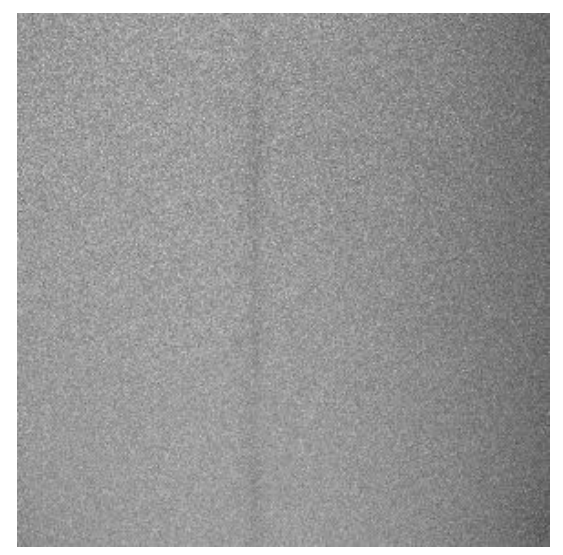

(c) $\mathrm{a}=0.6 \mathrm{~mm}, \varepsilon_{\mathrm{s}}=63 \mu \mathrm{m}$

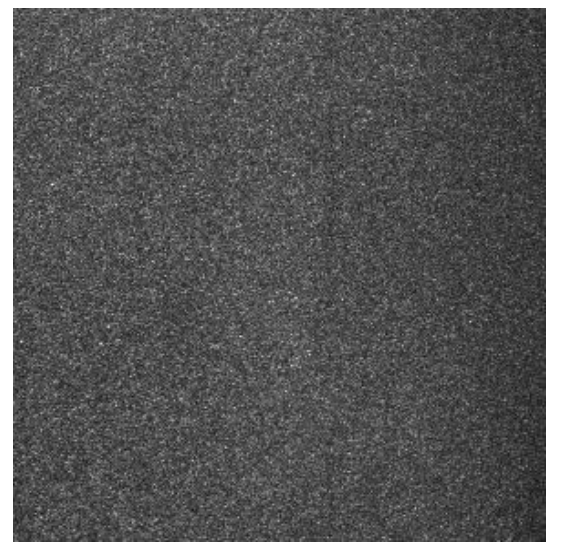

(b) $\mathrm{a}=7 \mathrm{~mm}, \varepsilon_{\mathrm{s}}=5 \mu \mathrm{m}$

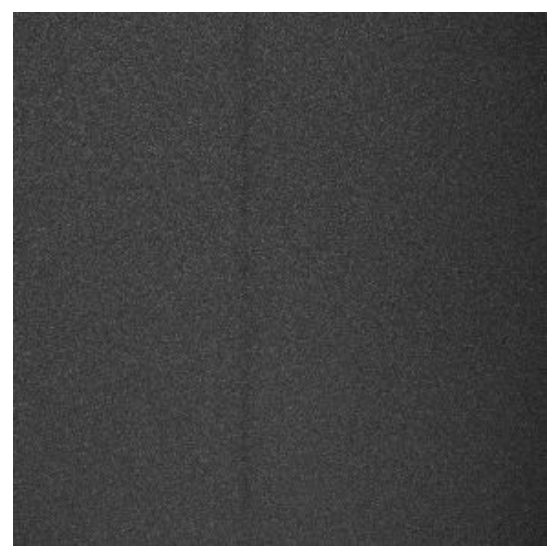

(d) $\mathrm{a}=0.2 \mathrm{~mm}, \varepsilon_{\mathrm{s}}=190 \mu \mathrm{m}$

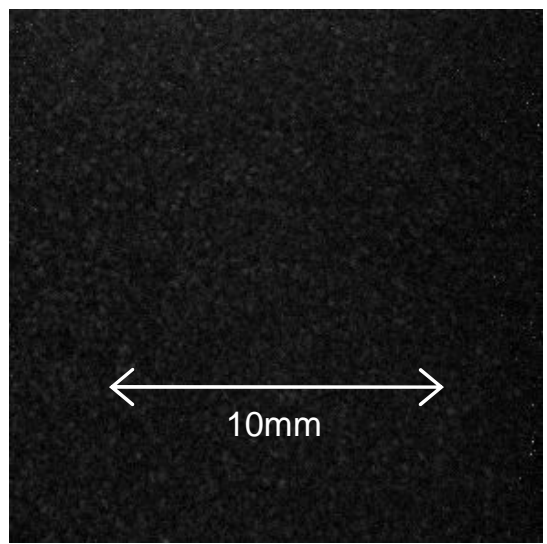

Figure 4. Images of subjective speckle taken through an open port in a 50mm diameter integrating sphere using a $633 \mathrm{~nm}$ HeNe laser and a CCD camera. A construction feature (the join between two hemispheres) is visible.

To understand how the speckle uncertainty could affect gas measurements, we replaced the camera with a photodetector (Thorlabs PD400) at a distance of $50 \mathrm{~mm}$ from the lens and illuminated the integrating sphere using our $1651 \mathrm{~nm}$ DFB laser. 2f-demodulated signals were recorded while the laser injection current was ramped in the range 40-70mA, corresponding to a sweep of approximately $21 \mathrm{GHz}$ or $0.2 \mathrm{~nm}$. Spectra were recorded for a $10 \mathrm{~mm}$ and $1 \mathrm{~mm}$ lens aperture. The results in Figure 5 have been normalised to account for differences in the mean recorded light intensity for each aperture. The standard deviation in the baseline was recorded as $0.13 \times 10^{-3}$ and $0.68 \times 10^{-3}$ respectively, showing a reduction in speckle noise by a factor of 5 when the aperture was increased by a factor of 10 . This is smaller than the 10 -fold improvement that we would have expected from equation (5) of Part 1, but nevertheless shows a considerable reduction. 


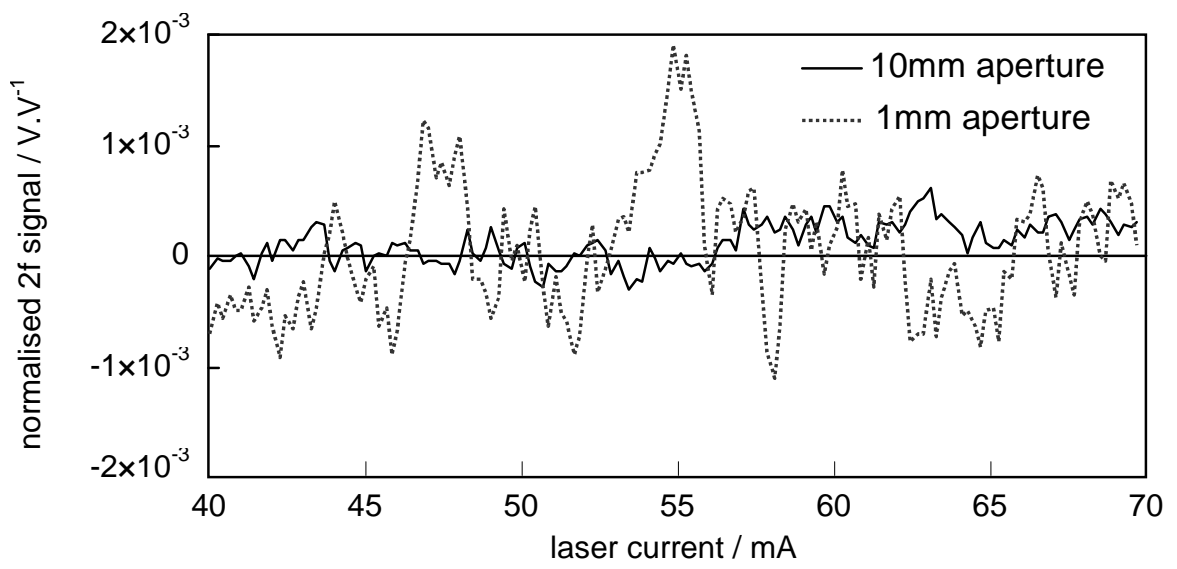

Figure 5. 2f-demodulated signals, normalised for mean recorded intensity, showing a reduction in subjective speckle noise following the use of a larger lens aperture.

\subsection{Self-mixing interference}

The laser diode was configured with a monitor photodiode at the rear facet in order to record the total laser diode output; we have previously found that this is a robust diagnostic test for self-mixing interference fringes as opposed to etalon-generated fringes ${ }^{[23]}$. Using the $50 \mathrm{~mm}$ diameter sphere, the exit aperture of the laser diode package was located $100 \mathrm{~mm}$ from the sphere first strike spot and for simplicity the window at the entrance aperture was removed, as shown in Figure 6.

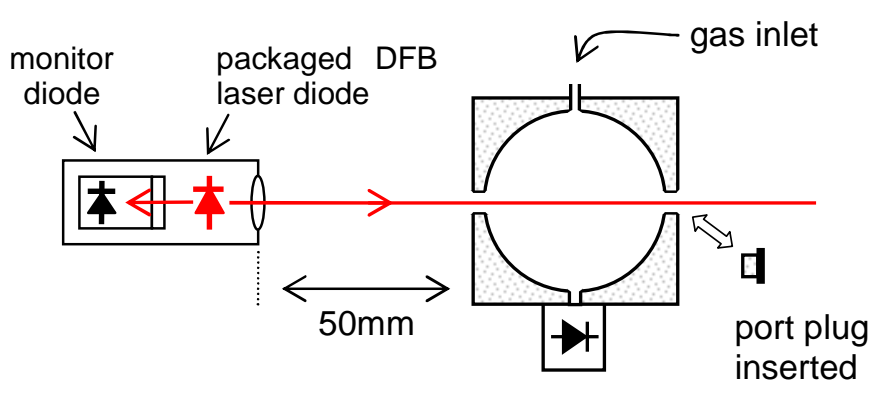

Figure 6. Experiment to measure the effects of feedback from the first strike spot to the laser diode.

2f-demodulated signals from the monitor diode were recorded while the DC laser diode current was ramped from 35 to $70 \mathrm{~mA}$. The results in Figure 7 show the generation of a significant, detectable interference fringe whose free spectral range is consistent with the round-trip cavity length from laser to 
sphere surface. In a control test, a port plug at the first strike spot was removed to allow the light to pass through the sphere. The results of this test, also shown in Figure 7, show a broader background fringe caused by the reference monitor photodiode itself; we have previously observed such fringes in more extensive self-mixing tests ${ }^{[23]}$. All our other experiments were conducted without the use of a monitor photodiode at the rear facet, in order to reduce this background fringe.

Our DFB laser diode is particularly susceptible to self-mixing interference because of its use of a large collimating lens, which acts to collect a greater proportion of backscattered light from the diffusely reflecting surface and deliver it to the laser diode. It is therefore possible that a reduced fringe amplitude would result from a better laser design, and from the use of balanced detection. With our apparatus, for the purpose of establishing limits of detection in the absence of self-mixing, we chose to simply place the laser diode a considerable distance from the sphere $(1 \mathrm{~m})$ in subsequent experiments. This reduces the fringes to a negligible level because of two effects: (i) a decrease in the intensity of backscattered light reaching the laser diode, and (ii) a reduction in the free spectral range of the fringes, which in a WMS experiment reduces their apparent magnitude ${ }^{[23]}$. 


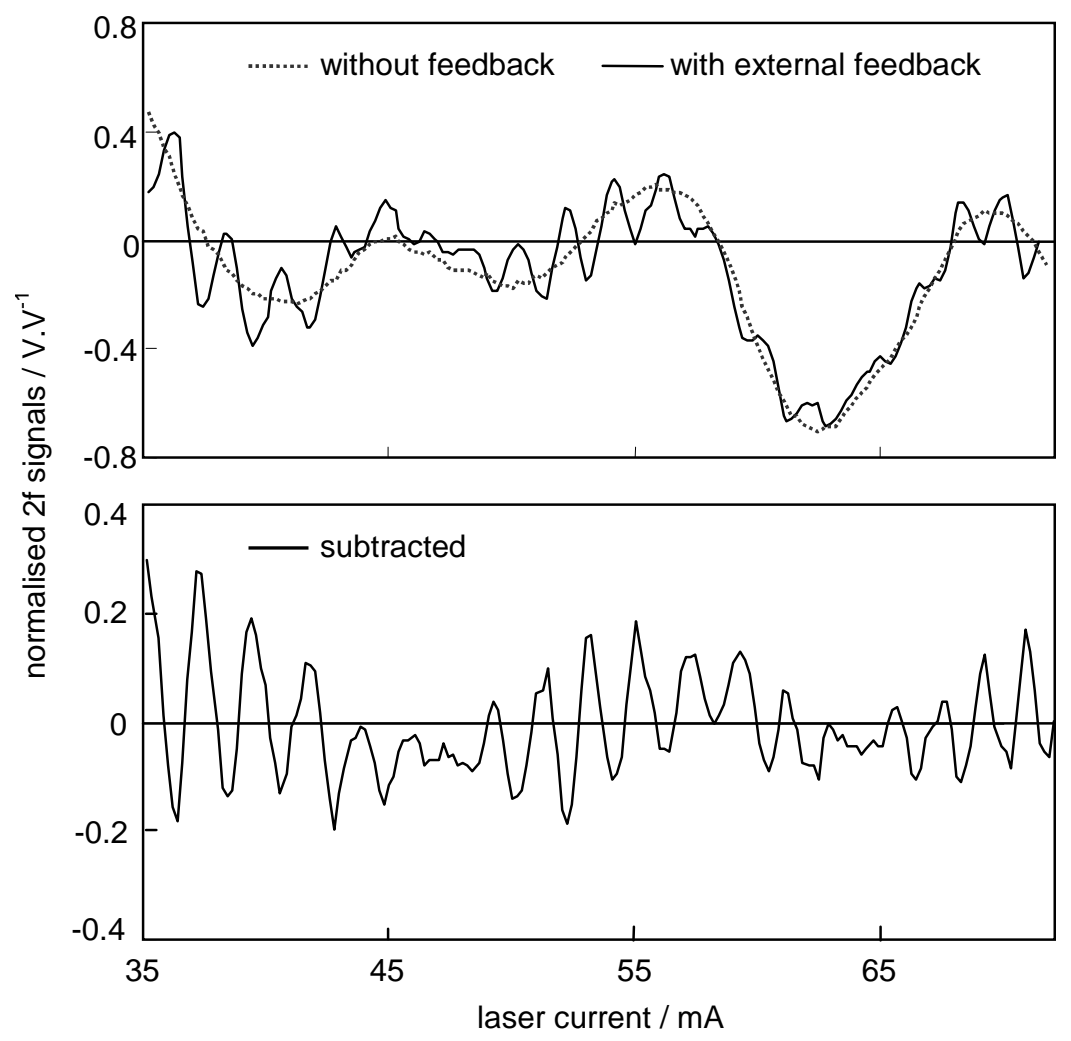

(a)

(b)

Figure 7. Signals from a monitor photodiode located at the laser diode rear facet to identify self-mixing interference. (a) Results of experiment with and without feedback from a 50mm integrating sphere. (b) Subtracted signals showing self-mixing fringes caused by feedback from the sphere.

\subsection{Results for gas detection}

Firstly, for the $50 \mathrm{~mm}$ diameter sphere, we recorded a direct scan of a gas line without implementing WMS. Because of reduced signal to noise ratios with the direct scan, a higher concentration of $5770 \mathrm{ppm}$ was used in this experiment. Figure 8 shows the resulting background scan and gas line scan. A baseline analysis yields a deviation from a flat baseline $(1 \sigma)$ at any single point in the scan of $70 \mathrm{ppm}$ or an uncertainty in $\Delta \Phi / \Phi_{0}$ of around $3 \times 10^{-3}$, which is close to our predicted value of $2 \times 10^{-3}$ in Table 1 . A similar level of performance might also be expected using direct spectroscopy with a conventional transmission cell. 


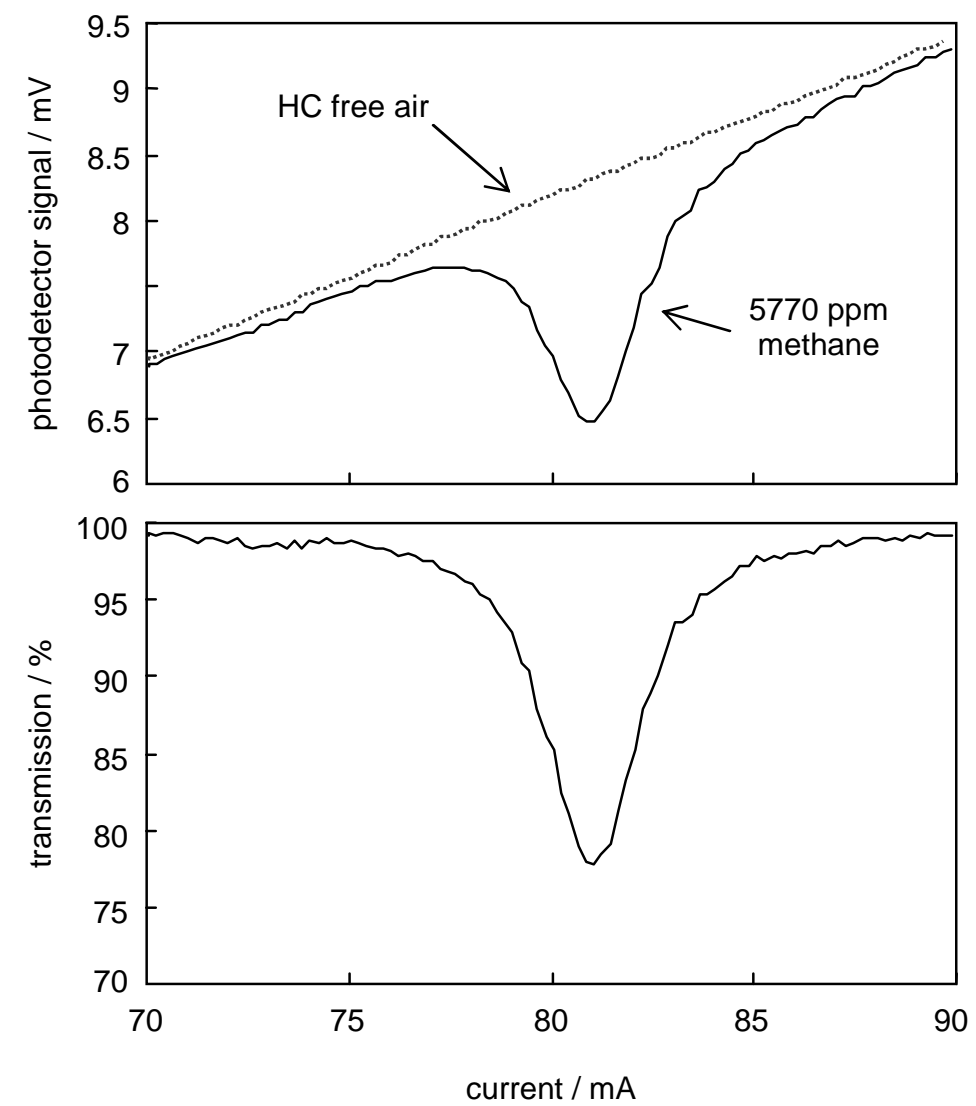

(a)

(b)

Figure 8. Direct spectral scan through a methane line at $1651 \mathrm{~nm}$, using a 50mm diameter integrating sphere. (a) Raw data, (b) calculated transmission.

Secondly, we implemented WMS and repeated the spectral scan. Using the 50mm diameter sphere, 2fdemodulated signals were recorded while the dc current was ramped from 40 to $75 \mathrm{~mA}$, giving a scan across the methane line of width $22 \mathrm{GHz}$ or $0.2 \mathrm{~nm}$. The lock-in amplifier time constant was set to $1 \mathrm{~s}$ to enable comparison with later results, and data points were recorded every $2 \mathrm{~s}$. This resulted in some slight loss of spectral resolution, but enabled scans to be completed within a few minutes. The results are shown in Figure 9. Analysis of the baseline yields an RMS deviation ( $1 \sigma$ ) noise equivalent absorbance (NEA) of around $10^{-4}$ (1.6 ppm for methane). For a WMS dither of $0.025 \mathrm{~nm}$, we would expect the intensity uncertainty $\Delta \Phi / \Phi_{0}$ to be between $2 \times 10^{-3}$ and $2 \times 10^{-7}$, with a corresponding NEA (via equation (3) in Part 1) of between $3 \times 10^{-3}$ and $3 \times 10^{-7}$. 


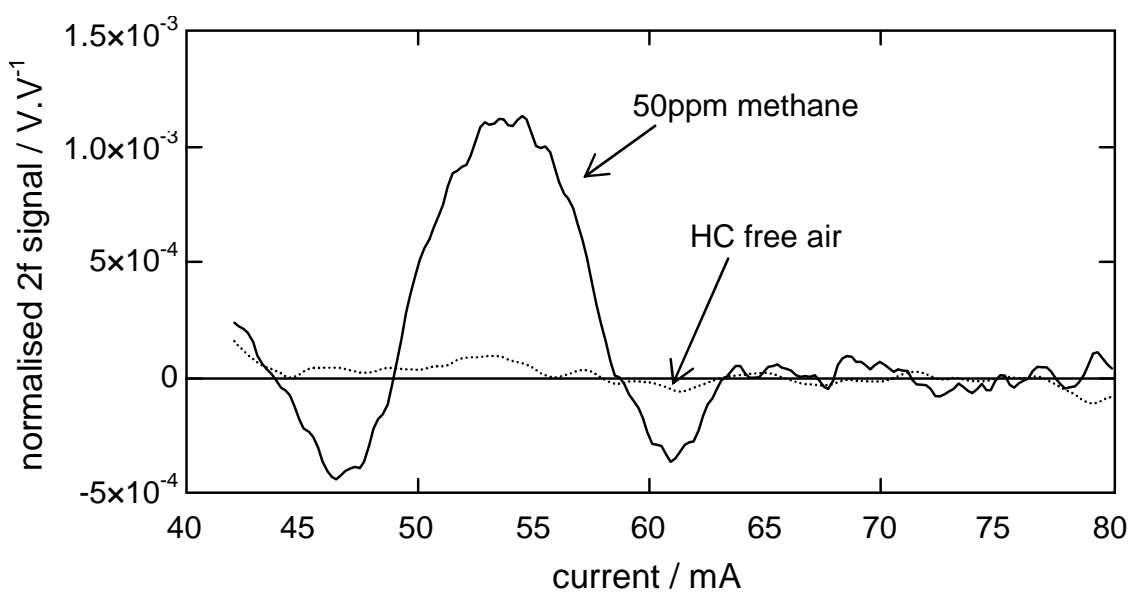

Figure 9. 2f-demodulated signals taken using a 50mm diameter sphere containing $50 \mathrm{ppm}$ methane and hydrocarbon free air.

In a final experiment the laser emission was locked to the peak of the absorption line. The $2 \mathrm{f}$-demodulated signal was recorded for a series of different concentrations of methane in air using the lock-in amplifier with a time constant of $1 \mathrm{~s}$. The results are shown for both the $50 \mathrm{~mm}$ and $100 \mathrm{~mm}$ spheres in Figure 10. Their effective pathlengths at low levels of absorption were established to be $1.2 \mathrm{~m}$ and $3.4 \mathrm{~m}$ respectively. This calibration followed the methodology established in reference [21], using equation (3) to establish the value of $\rho$ with high precision, then applying equation (2) which is valid at low absorption. Normalised $2 \mathrm{f}$ signals from the $100 \mathrm{~mm}$ sphere were approximately $3 \times$ those from the $50 \mathrm{~mm}$ sphere, in line with the pathlength ratio of 2.8 . 


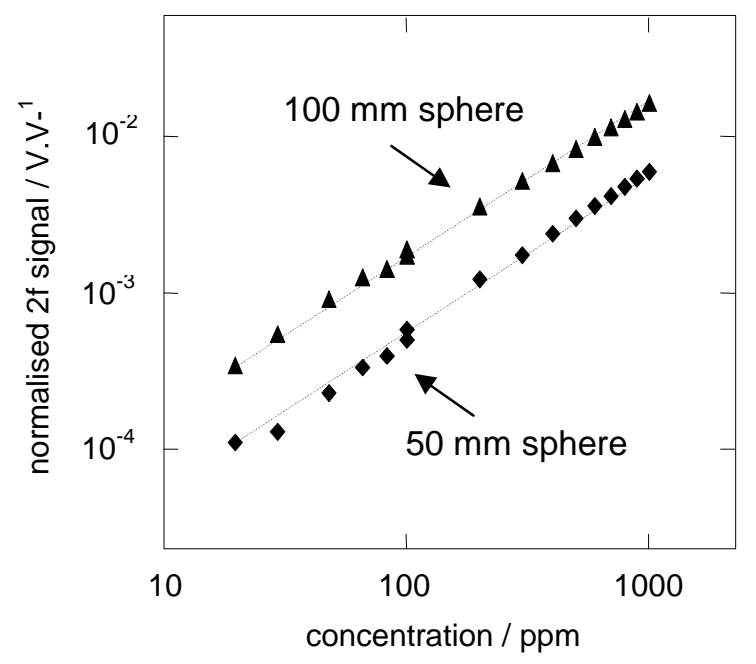

Figure 10. Normalised 2f-demodulated signals taken for different methane concentrations measurements in a $50 \mathrm{~mm}$ diameter and a $100 \mathrm{~mm}$ diameter sphere. The dashed lines show linear responses.

Limits of detection were established by taking the RMS deviation of a baseline measurement $(1 \sigma)$ over several periods of $1 \mathrm{~min}$, and normalising to a standard gas measurement. This yielded limits of detection $(1 \sigma)$ of $1 \mathrm{ppm}$ for the $50 \mathrm{~mm}$ sphere and $0.4 \mathrm{ppm}$ for the $100 \mathrm{~mm}$ sphere, translating to NEAs of around $5 \times 10^{-5}$ for both cases. We also measured an optical throughput of $0.3 \%$ for the $50 \mathrm{~mm}$ sphere (corresponding to the ratio of detected power to input power). Despite this low figure, the loss of light was not performance limiting.

\subsection{Assessment of drift}

The apparatus in Figure 2 was adapted along the lines of Figure 6 in Part 1, so as to include a second beamsplitter taking a proportion (approximately 45\%) of the light to a reference detector. This reference channel employed the same model of detector as the main signal channel and the same lock-in amplifier, with an identical set-up. The level of system drift was assessed by continuously recording data (with no gas in the cell) over a 21 hour period, for both the signal channel and the reference channel. Allan variance plots $\left(\sigma^{2}\right.$ as defined by Werle et al. $\left.{ }^{[24]}\right)$ were calculated for the recorded $2 \mathrm{f}$ measurements for each channel, using data normalised by division by the DC signals. These plots are shown in Figure 11 and, as in Part 1, describe a system dominated by drift. 


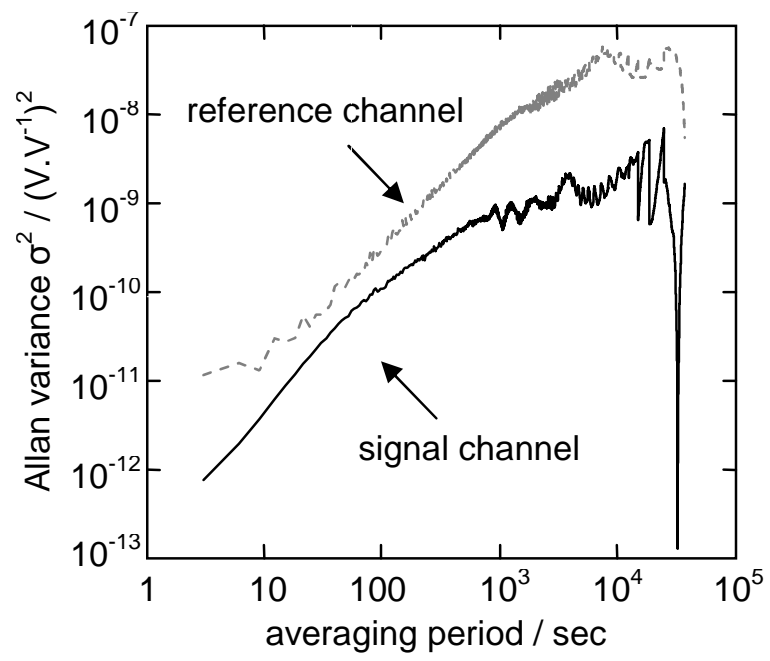

Figure 11. Allan variance plot for normalised $2 \mathrm{f}$ time series data taken over a 21 hour period with an empty cell.

The system drift appears to reach a maximum level of $\sqrt{ }\left(\sigma^{2}\right)$ corresponding to an NEA of $4 \times 10^{-4}$ for the integrating sphere and $1 \times 10^{-3}$ for the reference channel. As we found in Part 1 , the reference channel consistently drifts by more than the signal channel (this effect has been found to be repeatable). It may be that our signal channel has a larger and more representative sample of the laser diode output, and that the additional drift in the reference channel is cause by it sampling only a small portion of the beam. Or, our pellicle beamsplitter may be unstable over long timescales, with small movements affecting the reflected reference beam more than the transmitted signal beam.

\section{$5 \quad$ Discussion and conclusions}

We expect that random laser speckle is the fundamental performance limiting effect for integrating spheres. For direct spectral scans, our results have deviations from a flat baseline of $\Delta \Phi / \Phi_{0}=3 \times 10^{-3}$, which is of a similar magnitude to the level predicted by our theory $\left(2 \times 10^{-3}\right)$. For WMS, our results yield short-term limits of detection $(1 \sigma)$ of $1 \mathrm{ppm}$ for the $50 \mathrm{~mm}$ sphere and $0.4 \mathrm{ppm}$ for the $100 \mathrm{~mm}$ sphere, or NEAs of around $5 \times 10^{-5}$ in both cases. Longer-term drift was found to be at an NEA of $4 \times 10^{-4}$ for the $50 \mathrm{~mm}$ sphere. These figures fall within our expected range, however that range is too broad to act as confirmation of the theory laid out in Part 1 . We expected that speckle-related uncertainty in $\Delta \Phi / \Phi_{0}$ would be identical for spheres of different diameter. 
The main uncertainty in the theory lies in the value of $h$, the mean optical path deviation for the surface, which is determined by characteristics of the surface material and not quantified for the Spectralon or Zenith PTFE scatterers used in our integrating spheres. Assuming our longer-term drift figure of $4 \times 10^{-4}$ is at or above the level set by equation (9) in Part 1, we can set an upper limit for $h$ at around 10mm. This is rather large compared to the figure used for the diffuse optic in Part $1(6 \mu \mathrm{m})$, but is on a scale commensurate with the penetration depth of light into the reflective material. Further work is needed to investigate this parameter and to test the theory rigorously in this application, as well as to determine whether there are other sources of drift in our system that could be dominating the results.

The question then arises, whether there is scope to improve on the limits of detection achieved here. Firstly, for the purpose of our experiments we required integrating spheres that could be flexibly reconfigured, and used apertures large enough to permit capture of the subjective speckle field. The equivalent port area was therefore larger than could be achieved through careful design, and in particular the detector port area and the entrance aperture could be reduced in size. It may be possible to reduce the detector port to $2 \mathrm{~mm}$ diameter and the entrance aperture to $3 \mathrm{~mm}$ diameter. This would have the effect of increasing the mean reflectivity of the inner surface of the sphere, by reducing the non-reflective area $\left(f_{\text {total }}\right.$ in equation (4)), and thereby increasing the pathlength. Using equation (2), we anticipate that mean pathlengths could then be improved from 1.2 and $3.4 \mathrm{~m}$ to approximately $2.8 \mathrm{~m}$ and $6.5 \mathrm{~m}$ respectively for the $50 \mathrm{~mm}$ and $10 \mathrm{~mm}$ diameter spheres, improving limits of detection by a factor of approximately 2 in both cases. These pathlength multipliers do not compete with the best alternative multipass cells, but nevertheless offer a considerable improvement over single pass cell.

Secondly, we believe that residual drift effects may persist in our laser diode package, even after removal of the internal reference diode used in section 4.2. Previous work has shown that the use of a simple balanced detection scheme can improve the resulting uncertainties by a factor of 10 or more ${ }^{[23]}$. It is not certain whether this would yield the same improvement to the overall limit of detection, or reveal underlying random speckle noise that we expect to act as the fundamental limit. For an integrating sphere geometry, the noise levels for objective and subjective speckle are both expected to be similar. In contrast with the simple transmission cells of Part 1, the detection aperture for objective speckle is very large, reducing the associated speckle noise to a level comparable with subjective speckle recorded using a high NA lens.

Thirdly, we know that commercial integrating spheres are designed to give perfectly even irradiance over the surface of the sphere, by using materials that exhibit near ideal Lambertian scattering. For gas 
detection, our need for simple alignment translates to even irradiance at the surface local to the detector, and constant scattering behaviour at the first strike spot. This may offer the potential for designs better suited to gas detection. There may also be scope to better understand the value and role of the parameter $h$ borrowed from speckle theory, and to reduce $h$ while maintaining high levels of diffuse scatter.

We have confirmed the findings of other workers in this field, namely that integrating spheres are very simple to align, and that etalons are disrupted within the cell as long as there is no direct line of sight from the entrance aperture to the detector. We have also found it straightforward to avoid etalons between the entrance aperture window and other "upstream" optics. However, we have discovered that self-mixing or feedback interference can be performance limiting for lasers placed close to the integrating sphere, and optical feedback must therefore be reduced to realise low gas detection limits. Finally, although integrating spheres have very low levels of optical throughput ( $\sim .3 \%$ for the $50 \mathrm{~mm}$ sphere), this has not limited performance for the near-infrared InGaAs detectors that we used.

We can compare the performance of single pass cells and integrating spheres. In Part 1, using a single pass cell with a diffuse transmitter, we demonstrated a short-term NEA $(1 \sigma)$ of $2 \times 10^{-5}$, and longer-term drift of up to $3 \times 10^{-4}$ over 22 hours. Here, our short-term NEA was around $5 \times 10^{-5}$ and our long-term drift around $4 \times 10^{-4}$. For the singly scattering diffuser used in Part 1 , the value of $h$ was easier to estimate, and indeed it would be possible to confirm this by direct measurement, whereas for the multiply scattering material used in the integrating sphere its value is unknown. For subjective speckle with the same detection aperture and the same material properties, we would expect the level of speckle uncertainty to be similar for (i) a single pass cell of length $\mathrm{L}$, and (ii) an integrating sphere of diameter L, with the latter simply having a further $\sqrt{ } 2$ improvement as a result of depolarisation. For objective speckle, the latter has a wider field of view, reducing the expected speckle uncertainty compared to the single pass cell. For both objective and subjective speckle, the limit of detection for the integrating sphere then benefits from its longer pathlength, by a factor of between 20 and 50 depending on how the sphere has been configured (24 and 34 in our experiments, for the $50 \mathrm{~mm}$ and $100 \mathrm{~mm}$ spheres respectively).

To conclude, we have studied the effects of random laser speckle and self-mixing interference on TDLS based gas measurements made using integrating spheres. Experiments were conducted at $1651 \mathrm{~nm}$ for detection of methane, but the results can be translated to other wavelengths in the near-infrared. For a direct scan using a 50mm diameter commercial integrating sphere, the estimated short-term NEA $(1 \sigma)$ was $3 \times 10^{-3}$ or $75 \mathrm{ppm}$ methane. Using wavelength modulation spectroscopy reduced the short-term NEA to around $5 \times 10^{-5}$, corresponding to $1.2 \mathrm{ppm}$ and $0.4 \mathrm{ppm}$ respectively for sphere diameters of $50 \mathrm{~mm}$ and 
$100 \mathrm{~mm}$. Long-term drift was estimated to be at an NEA of $4 \times 10^{-4}$ for the $50 \mathrm{~mm}$ sphere, using WMS. We believe there may be scope to improve on these limits in future work.

\section{Acknowledgements}

This work was carried out under an EPSRC research grant (GR/T04601/01). Jane Hodgkinson is supported by an EPSRC Advanced Research Fellowship (GR/T04595/01). We would like to thank Dan Francis for his help with our data acquisition software.

\section{References}

[1] J.U. White, J. Opt. Soc. Am. 32, 285 (1942)

[2] D.R. Herriott, H. Kogelnik, R. Kompfner, Appl. Opt. 3, 523 (1964)

[3] S.M. Chernin, E.G. Barskaya, Appl. Opt. 30 (1), 51-58 (1991)

[4] Toptica GmbH, Product specification. Compact Herriott cell for absorption spectroscopy : CMP-30. Available at www.toptica.com, 2009.

[5] R Engeln, G Berden, R Peeters, G Meijer, Rev. Sci. Instrum. 69, 3763-3769 (1998)

[6] A. O’Keefe, D.A.G. Deacon, Rev. Sci. Instrum. 59, 2544-2551 (1988)

[7] A.G. Berezin, S.M. Chernin, D.B Stavrovskii. Proc $7^{\text {th }}$ International Conference on Tunable Diode Laser spectroscopy - TDLS09, Paper E2 (2009)

[8] H. I. Schiff, G. I. Mackay, J. Bechara, Chapter 5 in Air Monitoring by Spectroscopic Techniques, M W Sigrist, Ed, M Wiley, New York (1994)

[9] Labsphere Inc. "A guide to integrating sphere theory and applications," Labsphere, North Sutton, NH, USA (1998).

[10] P. Elterman, Appl. Opt. 9 (9), 2140-2142 (1970)

[11] L.M. Hanssen, K.A. Snail. Integrating spheres for mid- and near-infrared reflection spectroscopy., in Handbook of Vibrational Spectroscopy, vol 2, J.M. Chalmers, P.R. Griffiths, Eds, (Wiley, Chichester) pp 1175-1191, (2002)

[12] E.S. Fry, G.W. Kattawar, R.M. Pope, Appl. Opt. 31 (12), 2055-2065 (1992)

[13] I. Fecht, M. Johnson, Meas. Sci. Technol. 10 612-618 (1999)

[14] J. Hodgkinson, M. Johnson, J.P. Dakin, Appl. Opt. 44, 4360-4367 (2005).

[15] E. Hawe, P Chambers, C. Fitzpatrick, E. Lewis, Meas. Sci. Technol. 18, 3187-3194 (2007).

[16] E. Hawe, C. Fitzpatrick, P. Chambers, G. Dooly, E. Lewis, Sensor. Actuat. A 141, 414-421 (2008). 
[17] C.G. Venkatesh, R.S. Eng, A.W. Mantz, Appl. Opt. 19 (10) 1704-1710 (1980).

[18] R.M. Abdullin, A.V. Lebedev, Sov. J. Opt. Technol. 55 (3) 139-41 (1988).

[19] S. Tranchart, I.H. Bachir, J.-L. Destombes, Appl. Opt. 35 (36), $7070-7074$ (1996).

[20] D. Masiyano, J. Hodgkinson, R. P. Tatam, Applied Physics B 90, 279-288 (2008).

[21] J. Hodgkinson, D. Masiyano, R. P. Tatam, Applied Optics 48 (30), 5748-5758 (2009).

[22] A. Bozeit, J. Burke, H. Helmers, H. Sagehorn, R. Schuh. Opt. Laser Technol. 30, 325-329 (1998).

[23] D. Masiyano, J. Hodgkinson, S. Schilt, R. P. Tatam, Appl. Phys. B 96 (4), 863 (2009)

[24] P. Werle, R. Mücke, F. Slemr. Appl.Phys. B 57, 131-139, 1993. 